\title{
Nouvelles approches d'étude des réseaux microbiens
}

\author{
M. Bianchi ${ }^{1}$
}

Mots-clés : réseaux microbiens, techniques d'études, comptages bactériens, survie, résistance aux stress, distribution spécifique et diversité.

Les écosystèmes aquatiques sont des milieux privilégiés pour les transports d'organismes, les échanges entre individus, les évolutions rapides de communautés, combinés à la propagation rapide de signaux physiques et chimiques dans l'environnement. Les réseaux microbiens, composés d'une grande variété de micro-organismes (des bactéries au phytoplancton et aux protozoaires) dans divers états physiologiques, sont la base du fonctionnement de ces écosystèmes. L'identification des micro-organismes présents, et de leurs activités in situ, sont des défis technologiques permanents pour le microbiologiste.

Les différentes évolutions technologiques concernent : i) L'amélioration des techniques de dénombrement des bactéries vivantes. ii) L'adaptation des techniques de cytométrie (en image et/ou en flux) associées à des marqueurs fluorescents au comptage des micro-organismes in situ. Ces comptages peuvent concerner la présence d'une espèce ou d'une fonction métabolique (association de l'immunofluorescence avec la microscopie ou la cytométrie de flux, ou encore utilisation de sondes phylogénétiques fluorescentes à différents niveaux taxonomiques). iii) La combinaison des techniques décrivant l'état et les fonctions des microflores (biomasse, production, vitesse et rendement de croissance, diverses activités métaboliques intervenant dans les cycles biogéochimiques et/ou les processus de pollution ou de bioremediation) avec des techniques de chimie analytique fine décrivant le milieu naturel (HPLC, chromatographie gazeuse, spectrométrie de masse). iv) La recherche au niveau cellulaire de la réponse bactérienne aux conditions de stress par la mise en évidence de systèmes protéiques nouveaux.

\section{New approaches to study microbial networks}

Keywords : microbial networks, methods of study, bacterial counts, survival and stress resistance, specific diversity.

Aquatic ecosystems are characterised by physical and chemical fluctuations and gradients as well as by the resulting transportation's of organisms, modifications of communities and exchanges between individuals. The microbial networks, from the phytoplankton to the bacteria and protozoa, all in various states of activity, are a major component for the functioning of the pelagic ecosystems. The measurement of in situ numbers and activities of the micro-organisms is a permanent challenge for the microbiologist. Recent advances in techniques and technologies has provided new concepts to study micro-organisms in natural aquatic environments. They concern i) the improvement of counts of viable bacteria, ii) the combination of cytometry techniques (image analysis and/or flux cytometry) with fluorescent markers (fluorochromes, fluorescent genetic probes) to determine species or metabolic function, iii) the use of radioactive compounds combined with techniques of fine chemistry like GC, HPLC, mass spectrometry to describe metabolic rates, interaction with the chemical environment, iv) the assessment of bacterial response to external stresses at the molecular level.

\section{Introduction}

Les caractéristiques principales des environnements aquatiques sont la présence de fluctuations rapides et la distribution sous forme de gradients des paramètres physiques et chimiques. Par ailleurs, ces écosystèmes sont en permanence le site de mouvements des masses

1. Microbiologie Marine CNRS/INSU UPR 223, Université de la Méditerranée, Campus de Luminy, Case 907, F-13288 Marseille, France. d'eau et d'échanges avec les écosystèmes environnants par le biais d'apports latéraux et éoliens et d'exports (Fig. 1). Les organismes qui constituent les réseaux microplanctoniques, sont de taille microscopique et souvent sous forme unicellulaire, ils sont ainsi directement soumis aux nombreux changements de leur milieu environnant ce qui implique une adaptation constante des individus et des communautés. Par ailleurs, ces micro-organismes sont l'objet de transports sur des distances variables, de rencontres et d'échanges avec d'autre individus. 


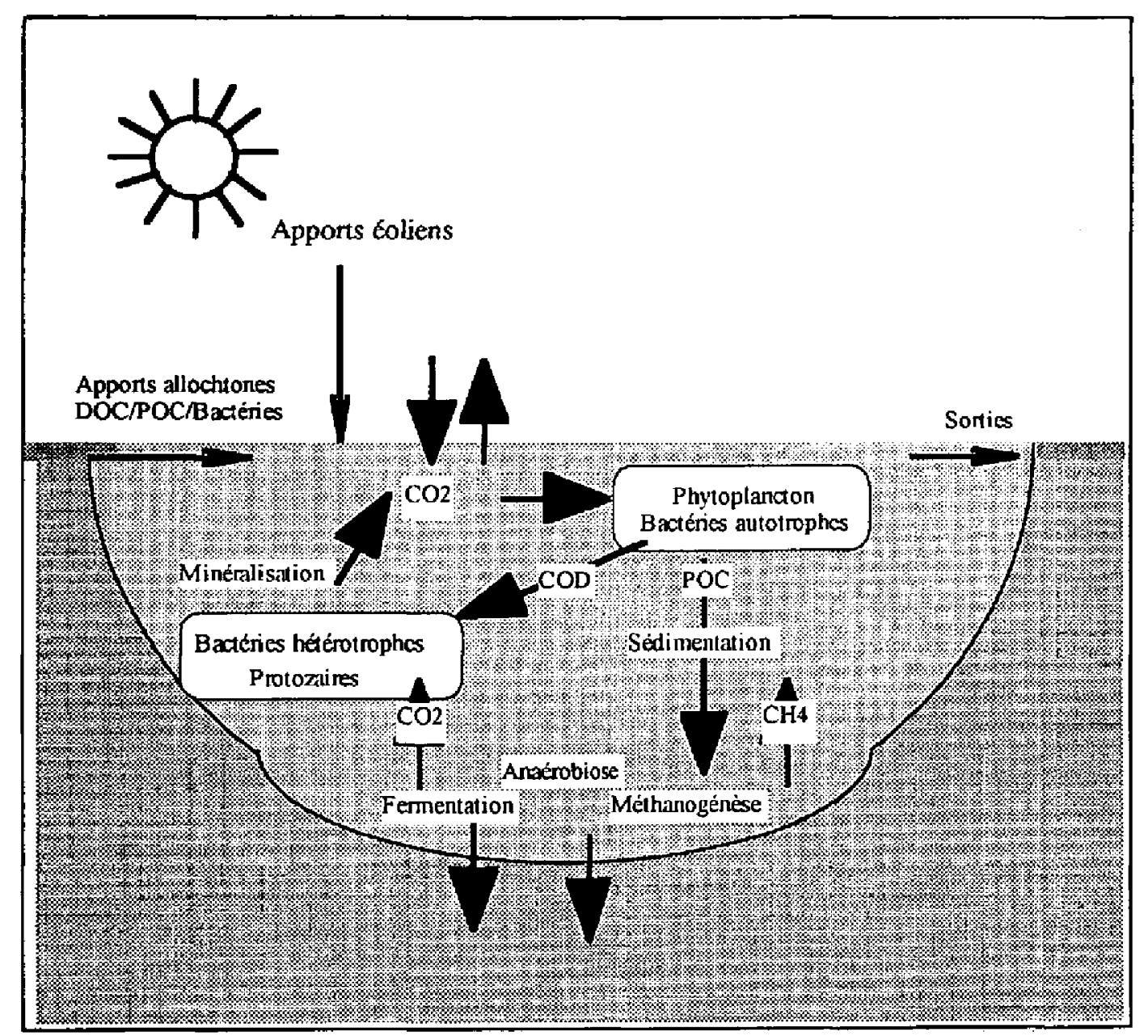

Fig. 1. Ecosystème aquatique et réseaux microplanctoniques.

Fig. 1. Aquatic ecosystem and microplanktonic networks.

Les micro-organismes composant les réseaux microbiens sont de deux types : les autotrophes comprenant les organismes photosynthétiques (phytoplancton, protozoaires chlorophylliens, cyanobactéries) et chimiosynthétiques (bactéries nitrifiantes par exemple) et les hétérotrophès (bactéries, protozoaires) aérobies et/ou anaérobies (Fig. 1). Les premiers sont à l'origine de la production de carbone organique «autochtone» dissous et particulaire, tandis que les micro-organismes hétérotrophes vont minéraliser la matière organique, y compris celle provenant des apports allochtones éventuels. La minéralisation du carbone peut être effectuée en conditions aérobies, le produit final étant le $\mathrm{CO}_{2}$ et/ou en anaérobiose le produit final pouvant être alors soit du $\mathrm{CO}_{2}$ soit du $\mathrm{CH}_{4}$.

Les micro-organismes et les communautés qu'ils constituent vont devoir faire montre de capacités d'adaptations pour survivre dans ces écosystèmes en perpétuelle évolution. Au sein du réseau microplanctonique (Fig. 2) les bactéries hétérotrophes jouent un rôle de premier plan dans la circulation de la matière organique, étant les seuls organismes capables d'utiliser la matière organique dissoute en concentration micro ou nanomolaire comme c'est souvent le cas en milieu aquatique. Cette réintégration de la matière organique dissoute dans le réseau trophique est bien connue sous le nom de «boucle microbienne» («microbial loop» : Azam et al. 1983). Au sein de cette boucle microbienne, les bactéries vont donc à la fois «limiter» les pertes en matière organique sous forme dissoute (MOD) en la transférant en partie vers les échelons supérieurs et, dans le même temps, effectuer une minéralisation de cette MOD. Ce processus va conduire à des pertes en carbone (sous forme de $\mathrm{CO}_{2}$ ) et à la remise à disposition des producteurs primaires de nutriments sous forme minérale.

L'ampleur du compartiment bactérien est régulée par deux facteurs qui sont i) la source de carbone et d'énergie permettant la croissance des cellules, et ii) la prédation par les protozoaires limitant la biomasse bactérienne. En dehors de zones de rejets à pollution bactérienne intense (tels les rejets d'égouts), un équilibre s'établit entre ces deux facteurs et le nombre total de bactéries fluctue peu dans les milieux aquatiques, demeurant compris entre $10^{5}$ et $10^{6}$ cellules par millilitre. 


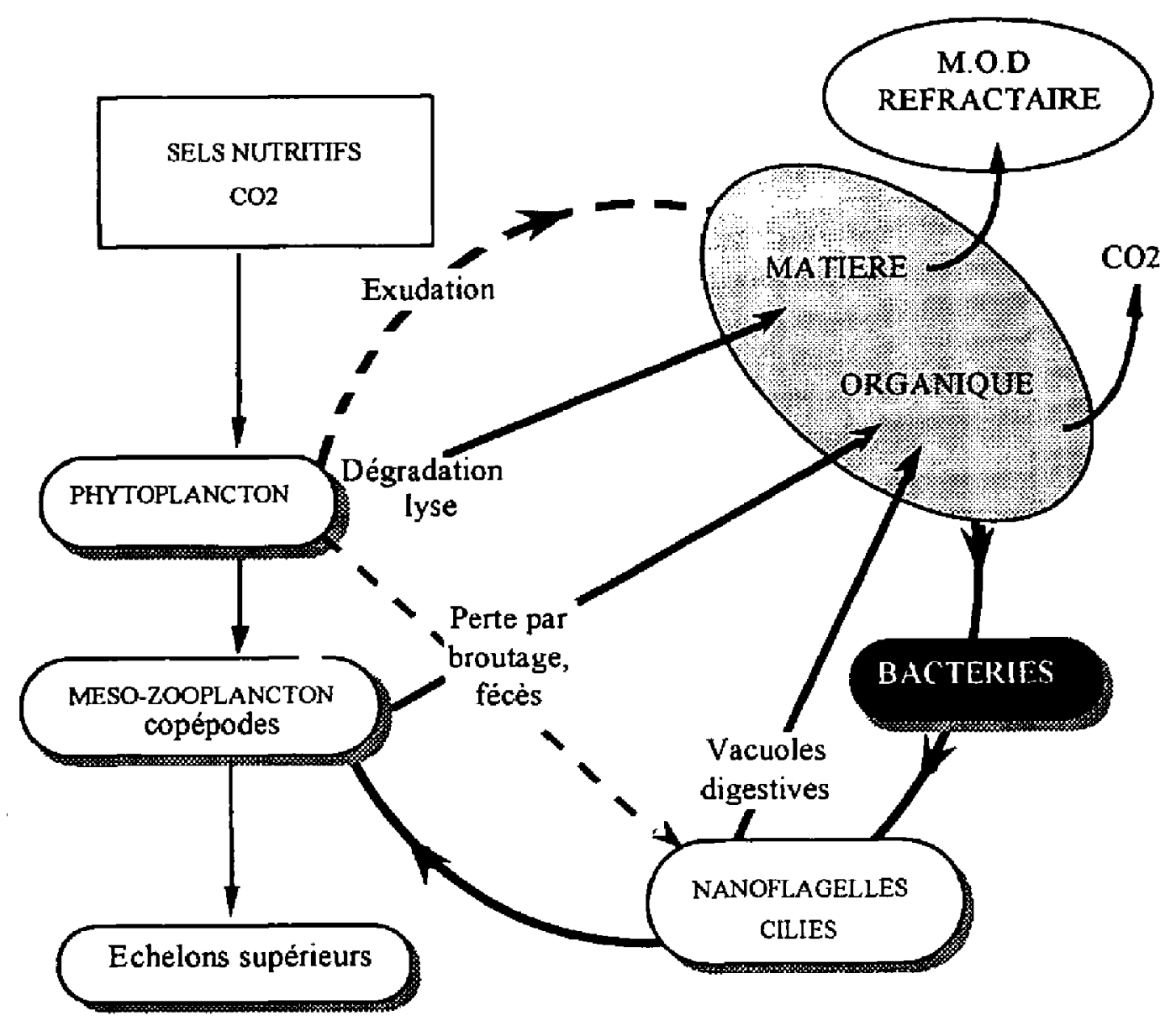

Fig. 2 Les bactéries dans les réseaux microplanctoniques.

Fig. 2. Bacteria in the microplanktonic networks.

Dans le milieu aquatique, la propagation des fluctuations des paramètres physiques et chimiques s'effectue sous forme de gradients plus ou moins brutaux. Ainsi, la fluctuation des paramètres qui vont influencer la croissance ou la mortalité des bactéries sera toujours (plus ou moins) graduelle. D'autre part, au niveau de la cellule bactérienne, le processus de limitation nutritive implique des changements séquentiels d'activités métaboliques, physiologiques et ultrastructuraux. De tels changements peuvent conduire à une réduction de la taille avec la production de minicellules (Morita 1985), à un changement dans les valeurs des constantes d'affinité $(\mathrm{Km})$ et de vitesse maximale (Vmax) d'utilisation des substrats (Nissen et al. 1984, Talbot \& Bianchi 1997) et enfin, à des changements d'état physiologique de la croissance vers la mortalité, la latence ou la survie (Fig. 3). Au niveau de la communauté bactérienne, comme dans toute communauté d'organismes vivants la diversité spécifique, la dominance de certaines espèces et l'abọndance relative des individus sont naturellement affectées par les modifications précédemment évoquées.

La compréhension et l'éventuelle prévision de la réponse des bactéries aux fluctuations de l'environnement, et inversement de l'impact des activités micro- biennes sur le fonctionnement de l'écosystème, nécessitent de prendre en compte un ensemble de questions qu'il est difficile de dissocier. Ces questions concernent :

- Le nombre de bactéries présentes dans un échantillon. Cette question qui paraît simple devient particulièrement difficile à résoudre lorsque l'on s'adresse à du matériel comme les agrégats en suspension dans la colonne d'eau au sein desquels il n'est pas toujours facile de compter les bactéries.

- La proportion de bactéries actives au sein d'un échantillon, c'est-à-dire : - combien de bactéries sont en phase de croissance active ; - combien sont en état de survie, (cette question peut concerner la totalité de la communauté ou une seule de ses composantes comme des bactéries indésirables rejetées dans le milieu par des activités humaines) ; - quelle proportion de la population réalise une activité fonctionnelle précise (comme la nutrification ou la dénitrification par exemple) dont l'impact sur l'écosystème souhaite être évalué et/ou contrôlé.

- Les facteurs et les processus qui régulent ces activités, qu'ils soient externes (température, sources de nutriments, prédation,...) et/ou intrinsèques (processus cellulaires mis en œuvre par les micro-organismes). 
Les substrats nutritifs et énergétiques

Sources ponctuelles dans le temps et dans l'espace

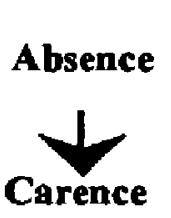

Quantité et qualité variables



$C, N, P, \ldots$

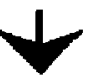

Processus de survie
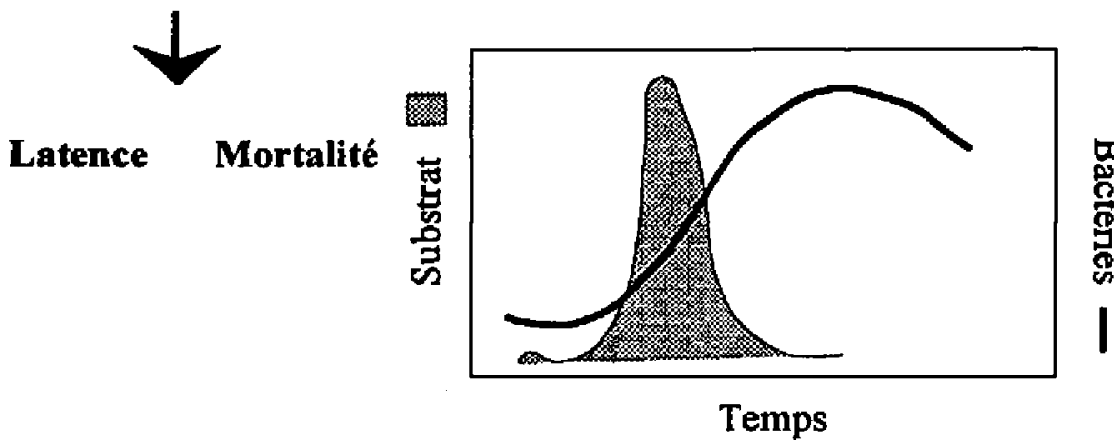

Présence
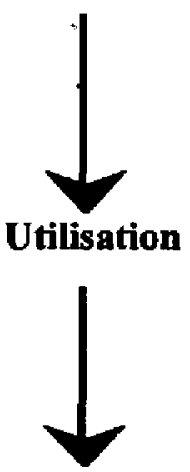

Croissance

Fig. 3 Les relations entre les bactéries et les substrats nutritionnels dans l'environnement naturel.

Fig. 3. Relationships between bacteria and nutrients in the natural environment.

- Les espèces bactériennes présentes dans les échantillons, en terme de diversité taxonomique et en terme d'abondance respective des différentes espèces. Ces deux questions sont tout à fait complémentaires puisqu'il s'agit de déterminer la variation du nombre d'espèces en fonction des conditions environnementales et de mettre en évidence la dominance quantitative d'individus traduisant leur meilleure adaptation aux conditions du moment.

Malgré la simplicité apparente de ces questions, les réponses à chacune d'elle représentent de véritables défis techniques pour les microbiologistes. La taille microscopique, l'insuffisance de critères morphologiques permettant une identification visuelle, la rapidité de changement d'état physiologique ne permettent pas une mesure directe (in situ) des paramètres bactériens. C'est à travers le recours à des techniques de plus en plus sophistiquées, basées sur l'utilisation d'appareillages complexes et onéreux, que l'on peut apprécier l'évolution de la profondeur et de la qualité des réponses apportées à ces différentes questions.

\section{L'amélioration des techniques de dé- nombrement de toutes les bactéries pré- sentes et des bactéries métaboliquement actives}

Pour mettre en évidence des «microbes» dont ils avaient deviné l'existence et l'importance, le génie des premiers microbiologistes a été «d'inventer» le milieu de culture, concept tout à fait étonnant dans le contexte de l'époque. Dès 1884 , certes, un pionnier de la microbiologie marine, a publié des dénombrements de bactéries sụr des échantillons d'eau ou de sédiment profonds, en utilisant des milieux de culture à base d'eau de mer stérile additionnée de bouillon de poulet ou de lait. Cette technique de dénombrement a prévalu pendant de nombreuses années bien que la réalité đes comptages ainsi effectués ait été de plus en plus contestée. En effet, il est apparu qu'il n'existe pas de milieu de culture universel et que seule une fraction souvent infime du peuplement global peut trouver des conditions favorables à leur prolifération dans un mi- 
lieu de culture quel qu'il soit. De plus, les bactéries aptes à croître sur le milieu choisi (ou disponible) n'étaient pas forcément celles réellement en activité dans l'environnement naturel.

En 1977, Hobbie et al. publient une méthode de comptage au microscope qui permet de visualiser toutes les bactéries présentes dans les échantillons, en utilisant un fluorochrome se fixant sur les acides nucléiques des cellules. La réalisation de cette technique a été rendue possible grâce i) à la microscopie à épifluorescence permettant l'observation sur le filtre de la lumière re-émise par le fluorochrome après excitation à une longueur d'onde adéquate, ii) l'utilisation de filtres en polycarbonate, offrant une surface parfaitement plane et permettant l'observation des cellules à leur surface, iii) la coloration par un fluorochrome sélectif. L'utilisation intensive de cette technique, avec des variantes dans la nature du fluorochrome, a permis de constater que l'écart entre les comptages de bactéries par mise en culture des échantillons et le dénombrement du nombre total de bactéries pouvait atteindre plusieurs ordres de grandeur. Il faut quand même souligner que dans les comptages dits «du nombre total de bactéries» il n'est encore pas possible de distinguer les bactéries réellement actives de celles en état de latence ou encore de déterminer l'appartenance spécifique des cellules observées. A partir de ces techniques de base, différentes variantes ont vu le jour, telles celles utilisant des anticorps fluorescents pour la reconnaissance d'espèces définies (Josserand \& Cleyet-Marel 1979).

Plusieurs techniques ont été développées pour la recherche des bactéries en état d'activité métabolique dans le milieu naturel. Ainsi, des méthodes de «culture» dans des milieux à base d'eau de mer de l'échantillon naturel, sans aucune adjonction d'éléments nutritifs, a permis de pettre en évidence les bactéries viables dans des conditions nutritionnelles proches de celles du milieu naturel, par le comptage en microscopie à épifluorescence, de la formation dans le temps de microcolonies (Bianchi \& Giuliano 1996). Une autre approche concerne la mise en évidence de l'activité respiratoire des bactéries par la mesure de l'activité des déshydrogénases et divers coenzymes qui forment la chaîne respiratoire (Houri-Davignon \& al. 1989). Le principe est basé sur la réduction d'un substrat oxydé par exemple un sel de tétrazolium : 2-para-iodophényl 3-para-nitrophényl 5-phényl tétrazolium chloride ou INT entrand en compétition avec l'accepteur terminal de la chaîne respiratoire (Trevors et al. 1982). Les échantillons sont incubés avec le substrat (INT) et le produit de la réduction (INT-Formazan) est extrait avec du méthanol puis dosé au spectrophotomètre à
$480 \mathrm{~nm}$ (Gammelgaard et al. 1992). Un comptage des bactéries métaboliquement actives utilise ce même principe, mais l'INT est remplacé par du CTC (5-cyano-2,3-ditolyl tetrazolium chloride), un accepteur terminal d'électrons qui produit un cristal fluorescent de CTC-formazan après réduction. Les cellules actives présentent donc une fluorescence qui permet de les détecter à une longueur d'onde de $590 \mathrm{~nm}$ après excitation du CTC-formazan à $540 \mathrm{~nm}$, les comptages s'effectuant en cytométrie en flux (Kapreylants \& Kell 1993).

\section{L'adaptation des techniques de cytomé- trie en image et/ou en flux au comptage des micro-organismes}

L'évolution la plus récente concerne l'introduction de la cytométrie en flux couplée au marquage des cellules par des fluorochromes ou par des marqueurs génétiques (sondes moléculaires). Ces techniques permettent de compter et d'identifier des cellules par leur fluorescence. A l'heure actuelle dans les milieux aquatiques, elles sont surtout utilisées pour les organismes photosynthétiques dont les pigments chlorophylliens constituent d'excellents marqueurs. Les premières applications de cette technique aux dénombrements bactériens ont concerné des bactéries présentant un rapport direct avec les actions humaines (Steen et al. 1982, Mansour et al. 1985). Depuis peu, cette technique a commencé à être appliquée aux comptages bactériens dans les échantillons naturels (Montfort \& Baleux 1992, Trousselier et al. 1993). Dans le milieu marin, l'utilisation de nouveaux coloränts (TOTO-1, TO-PRO-1, SYBR Green I) se fixant sur. les acides nucléiques et excités par une lumière bleue ou U.V. a permis de différencier les prokaryotes photosynthétiques, comme les Synechococcus et les Prochlorococcus, des prokaryotes hétérotrophes (Marie et al. 1997). Encore une fois, ce paragraphe n'a pas pour but une description exhaustive de toutes les avancées techniques récentes, mais.d'en démontrer le foisonnement et la diversité.

\section{La combinaison des techniques de mesu- re des activités bactériennes et de l'analyse du milieu naturel}

Les populations naturelles comportent des cellules dans des états physiologiques différents, il est pourtant nécessaire d'évaluer la production de carbone bactérien et les activités de minéralisation de la matière orga- 
nique qui vont conditionner les relations entre les bactéries et leur environnement. Les vitesses de croissance des bactéries planctoniques peuvent être estimées en mesurant l'incorporation dans les macromolécules de précurseurs radioactifs. Ainsi, la synthèse d'ADN (acide deoxyribonucléique) est mesurée par incorporation de $\left[{ }^{3} \mathrm{H}\right]$ thymidine (Furhman \& Azam 1982) tandis que la synthèse protéinique est démontrée par l'incorporation de $\left[{ }^{3} \mathrm{H}\right]$ leucine dans les protéines (Kirchman et al. 1985). Ces techniques ont été très largement utilisées ces dernières années en écologie bactérienne et ce, malgré l'inconvénient de la nécessité de facteurs de conversion pour convertir les vitesses d'incorporation du traceur (mesurée) en vitesse de synthèse de macromolecules. Ces facteurs étant difficiles voir impossibles à établir expérimentalement ce sont souvent des facteurs de conversion «théoriques» qui sont utilisés.

Par ailleurs, l'action des bactéries hétérotrophes sur la matière organique a fait, et fait toujours, l'objet de nombreuses mesures à travers deux principales étapes : l'hydrolyse des macromolécules ne pouvant pénétrer dans la cellule bactérienne et le prélèvement et l'incorporation des molécules de faible poids moléculaire. La première étape est déterminée à l'aide de la technique du substrat modèle fluorogène qui est un polymère contenant des molécules naturelles comme les acides aminés ou les sucres (Somville \& Billen 1983). L'hydrolyse de ce substrat sous l'action des enzymes bactériennes libère un composé fluorescent dont l'intensité est mesurée au spectrofluorimètre. L'utilisation des composés sous forme de monomères et/ou dimères est déterminée par l'incorporation de radiotraceurs (acides aminés, sucres, lipides) marqués au ${ }^{14} \mathrm{C}$ ou au ${ }^{3} \mathrm{H}$ dans les cellules (Wright \& Hobbie 1966). Ce sont des techniques qui permettent d'utiliser les traceurs à des concentrations proches de celles du milieu naturel, quand il est toutefois possible de déterminer les concentrations de ces composés.

En effet, pour re-situer et interpréter les processus bactériens et les vitesses à laquelle ils se réalisent, il est nécessaire de considérer la matière organique présente car elle conditionne la limitation par la ressource du compartiment bactérien. Cette matière organique se présente sous forme plus ou moins complexe, par la taille moléculaire, la labilité, la famille chimique des composés qui entrent en jeu. Autant de paramètres qui vont déterminer le développement des bactéries et réciproquement le temps de résidence et le devenir de la matière organique. Une synthèse des processus et des techniques d'étude est proposée par Bianchi et al. (1996).

\section{Biologie moléculaire et écologie bacté- rienne}

Durant les cinq dernières années, l'intégration des techniques de biologie moléculaire comme l'analyse des séquences d'ARNr $16 \mathrm{~S}$ et $23 \mathrm{~S}$ après amplification des gènes par la PCR (Polymerase Chain Reaction) ont eu pour conséquence une meilleure compréhension de la diversité phylogénétique, des bases moléculaires des adaptations physiologiques, de la transduction des signaux environnementaux et des réponses des microorganismes à ces signaux. La réponse à un stimulus peut être considérée à deux niveaux d'étude : les individus d'une même espèce ou l'ensemble des espèces d'une communauté.

\subsection{Etude des effets de la carence nutritionnelle sur les cellules bactériennes}

Parmi les changements de l'environnement que nous avons évoqué, les situations de carence sont extrêmement courantes pour les bactéries et ces phénomènes se présentent comme un exemple de résistance à un stress de ces micro-organismes. D'une part, en milieu aquatique naturel, il semblerait que les bactéries aient des systèmes multiples de transport de substrat énergétique et carboné (Azam \& Hodson 1981, Talbot \& Bianchi '1997), mais l'étude au niveau moléculaire de ces processus de transport multiphasiques reste à réaliser. D'autre part, il a été montré que les bactéries répondent aux changements environnementaux par le biais de systèmes à double composants : une protéine capteur perçoit des signaux physiologiques pertinents et phosphoryle un régulateur de réponse. La fonction du régulateur de réponse est souvent, mais pas toujours, de moduler la transcription d'un set donné de gènes (Parkinson 1993).

Si le milieu ambiant contient une source nutritionnelle, les cellules bactériennes se divisent. Par contre, lorsque le milieu est carencé ou ne contient plus un élément essentiel, les cellules entrent en phase stationnaire, phase qui induit des changements importants dans la physiologie et la morphologie cellulaire, une altération de la composition de la membrane et des différences dans l'enroulement et la compacité de l'ADN. En-dessous d'un certain seuil de concentration d'un élément majeur, la cellule va répondre en induisant des systèmes de transport à forte affinité pour le substrat et en mettant en place des voies métaboliques pour l'exploitation de sources alternatives du nitriment. Les systèmes de régulation impliquent le contrôle de larges groupes de gènes structuraux. 
Les effets des. carences en carbone, en azote, en phosphore et de ces trois éléments majeurs combinés sur une bactérie marine (Vibrio sp. 14) a été étudié par Nyström et al. (1992). Les stimulons de carence présentent un nombre équivalent de protéines (une vingtaine) pour chacun des éléments séparés, mais seulement 42 protéines sont induites lors de la carence combinée de ces trois éléments. Les cellules carencées en azote et phosphore démontrent un faible taux de survie et une faible résistance à d'autres stress comme la chaleur ou l'exposition aux U.V. Au contraire, la carence carbonée, tout comme la carence multiple, permettent une survie à long terme et une meilleure résistance aux autres stress. D'autre part, selon les différentes carences les stimulons ne sont pas identiques, par exemple 14 sur 42 des protéines induites lors de la carence multiple sont spécifiques à cette carence et $4 / 5$ des protéines induites lors de la carence en phosphate ne se retrouvent pas dans le stimulons de la carence multiple (Nyström et al. 1992). De plus, il a été mis en évidence qu'une cyanobactérie (Synechococcus sp. WH 7803) possède des «phosphate binding proteins» homologues à celles d' $E$. coli dont on connaît bien le Pho régulon qui inclue plusieurs gènes et opérons codant pour des protéines dans la prise et l'utilisation de sources extracellulaires de phosphore (Mann, 1995).

\subsection{Application des techniques de séquençages d'ARN, de la PCR et des sondes nucléiques}

Les microbiologistes de l'environnement, et en particulier du milieu aquatique, ont tout de suite perçu l'intérêt des méthodes faisant appel aux techniques s'adressant directement au matériel génétique pour identifier les bactéries in situ. Ainsi, dès 1990 Atlas \& Bej testaient l'amplification du matériel génétique des entérobactéries et les sondes nucléiques spécifiques pour leur connaissance. Le principe est relativement simple : à partir d'une espèce connue et cultivée, une cible (gène) est choisie et des amorces spécifiques encadrant le gène sont identifiées (Fig. 4). Après extraction de l'ADN total dans les échantillons naturels, une PCR est réalisée à l'aide des amorces spécifiques qui vont donc amplifier une certaine partie de l'ADN. Après clonage dans un plasmide il sera possible de déterminer si la souche (ou la fonction) choisie est présente ou non dans l'échantillon de départ.

Ces techniques très prometteuses nécessitent encore quelques améliorations qui sont d'ailleurs en cours (Wintzingerode et al. 1997). Par exemple le dénombrement spécifique d'espèces par «PCR quantitative» commence à apparaître. Ainsi, les gènes codant pour les deux processus importants du cycle de l'azote que sont la nitrification et la dénitrification font l'objet de recherches relativement nombreuses (revue de Ward 1995). Contrairement à la nitrification qui est réalisée par un petit nombre d'espèces bactériennes autotrophes, la dénitrification peut être le fait de nombreuses espèces de bactéries hétérotrophes sans lien taxonomique. Ce type de sondes moléculaires réalisées à partir d'un gène codant pour un enzyme présentent un grand intérêt en écologie microbienne en permettant des mesures in situ d'activités importantes pour le fonctionnement de l'écosystème tout en s'affranchissant de la nature taxonomique, et par là des besoins culturaux des bactéries concernées.

La composition taxonomique et la diversité spécifique des bactéries des milieux naturels ont été également été abordées par le biais de ces techniques. Une revue de ces études dans le domaine océanique a été faite par Giovannoni et al. (1995). Les premiers travaux montrent une grande diversité taxonomique des espèces en présence. Malheureusement aucune donnée n'est encore disponible sur l'abondance relative des espèces, critère tout à fait capital pour la compréhension d'une éventuelle succession de populations lors de changements des conditions environnementales.

Encore une fois, cet article n'a pas pour vocation une description exhaustive de toutes les techniques explorées à l'heure actuelle, mais de donner simplement un aperçu, par des exemples choisis, des possibilités qui s'ouvrent pour de nouvelles prospections dans le milieu naturel. Bien entendu, d'autres techniques sont proposées aux microbiologistes de l'environnement comme la comparaison des profils de RNA de faible poids moléculaire (Höflè 1992), technique qui nécessite cependant la mise en culture des micro-organismes, créant une sélection gênante des bactéries dès le départ de l'analyse.

La microbiologie des milieux aquatiques est une discipline en pleine évolution. Cette discipline a d'abord adapté et adopté les techniques qui ont permis la compréhension, au niveau cellulaire, des réactions des organismes aux variations de leur environnement. Aujourd'hui elle profite des dernières techniques permettant l'étude au niveau moléculaire des processus d'adaptation aux conditions environnementales. Ainsi, dans les années à venir, la compréhension du fonctionnement des écosystèmes pourra bénéficier des progrès attendus dans la définition de la diversité des communautés microbiennes, ainsi que des possibilités de mesure, à travers leur régulation génétique, de fonctions métaboliques majeures. 


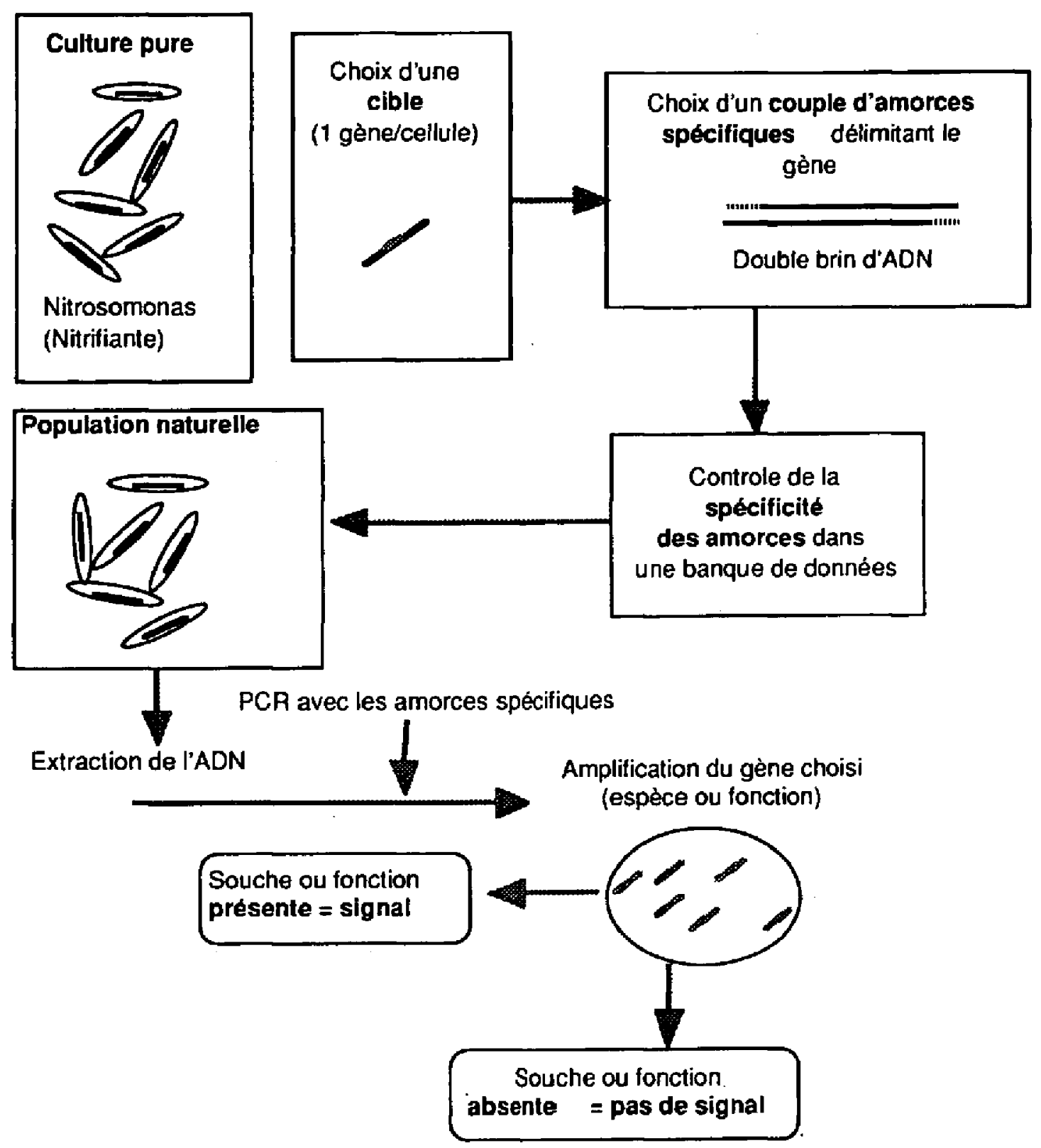

Fig. 4. Description schématique du protocole d'une reconnaissance sélective d'une espèce ou d'une fonction bactériennes par sonde nucléique.

Fig. 4. Description of the protocol for identification of a bacterial species or function by using a specific nucleic probe.

\section{Travaux cités}

Atlas R. \& Bej A. 1990. - Detecting bacterial pathogens in environmental water samples by using PCR and gene probes. In PCR Protocols. A guide to methods and applications. Innis MA, Gelfand DH, Sunsky JJ, White TJ (Eds), Academic Press, London : 399-406.

Azam F. \& Hodson R.E. 1981. — Multiphasic kinetics for D-glucose uptake by assemblages of natural marine bacteria. Mar. Ecol. Prog. Ser., $6: 213-222$.

Azam F., Fenchel T., Field J.G., Gray J.S., Meyer-Reil L.A. \& Thingstad F. 1983. - The ecological role of water-column microbes in the sea. Mar. Ecol. Prog. Ser., $10: 257-263$.
Bianchi M., Bianchi A., Goutx M., Marty D., Sempéré R. \& Van Wambeke F. 1996. - Degradation of organic matter by heterotrophic bacterial activity. In Integrated Marine System Analysis. J. Baeyens, F. Dehairs \& L. Goeyens (Eds). European Network for Integrated Marine System Analysis. Brugges 29/02/96-02/03/96. VUB.

Bianchi A. \& Giuliano L. 1996. - Enumeration of viable bacteria in the marine pelagic environment. Appl. Environ. Microbiol., 62 : 147-177.

Certes A. 1884. - Sur la culture à l'abri des germes atmosphériques, des eaux et des sédiments rapportés par les expéditions du «Travailleur» et du «Talisman» : 1882-1883. C. R. Hebd. Séances Acad. Sci.,98 : 690-693. 
Furhman J.A. \& Azam F. 1982. - Thymidine incorporation als i measure of heterotrophic bacterioplankton production in murine surface waters : evaluation and field results. Marine Biol., 66 : 109-120.

Gammelgaard A., Freeman C. \& Lock M.A. 1992. - Measurement of electron transport system activity in sphagnum-derived peat. Soil. Biol. Biochem., 25 : 505-507.

Giovannoni S.J., Mullins T.D. \& Field K.G. 1995. - Microbial diversity in oceanic systems : rRNA approaches to the study of unculturable microbes. In : Molecular ecology of aquatic microbes. J.E. Hobbie, R. Daley \& S. Jasper 1997. - Use of Nuclepore filters for counting bacteria by epifluorescence microscopy. Appl. Environ. Microbiol., 33 : 1225-1228.

Höfle M.G. 1992. - Aquatic microbial community structure and dynamics during large scale release of bacteria as revealed by low-molecular-weight RNA analysis. Appl. Environ. Microbiol., $58: 3387-3394$.

Houri-Davignon C., Relexans J.C. \& Etcheber H. 1989. - Measurement of actual electron transport system ETS. - acitivity in matine sediments by incubation with INT. Environ. Technol. Letters, $10: 91-100$.

Joint I. 1995. - Molecular ecology of aquatic microbes. NATO ASI Series, Series G : Ecological Sciences, vol 38. Springer-Verlag, Berlin Heidelberg : 217-248.

Josserand A. \& Cleyet-Marel J.C. 1979. - Isolation from soils of Nitrobacter and evidence for novel serotypes using immunofluorescence. Micro. Ecol., 5 : 197-205.

Kapreylants A.S. \& Kell D.B. - The use of 5-cyano-2,5-ditolyl tetrazolium chloride and flow cytometry for the visualisation of respiratory activity in individual cells of Micrococcus luteus. J. Microbiol. Meth., $17: 115-122$.

Kirchman D., K'nees E. \& Hodson R. 1985. — Leucine incorporation and its potential as a measure of protein synthesis by bacteria in natural aquatic systems. Appl. Environ. Microbiol., 49 : 599607.

Mann N. 1995. - How do cells express nutrient limitation at the molecular level ? In : Molecular ecology of aquatic microbes. Joint I (Ed.) - NATO ASI Series, Series G : Ecological Sciences, vol 38. Springer-Verlag, Berlin Heidelberg : 171-190.

Mansour J.D., Robson J.A., Annd C.W. \& Schulte T.H. 1985. Detection of Escherichia coli in blood using flow cytometry. $C y$ tometry, $6: 186-190$.
Marie D., Partensky F., Jacquet S. \& Vaulot D. 1997. - Enumeration and cell cycle analysis of natural populations of marine picoplankton by flow cytometry using the nucleic acid stain SYBR Green I. Appl. Environ. Microbiol., 63 : 186-193.

Montfort P. \& Baleux B. 1992. - Comparison of flow cytometry and epifluorescence microscopy for counting bacteria in aquatic ecosystems. Cytometry, $13: 188-192$.

Morita R.Y. 1985. - Starvation and miniaturisation of heterotrophs, with special emphasis on maintenance of the starved viable state. In «Bacteria in their natural environments» M.M. Fletcher \& G.D. Floodgate (Eds). Academic Press, London : 111-130.

Nissen H., Nissen P. \& Azam F. 1984. - Multiphasic uptake of Dglucose by an oligotrophic marine bacterium. Mar. Ecol. Prog. Ser., $16: 155-160$.

Nyström T., Olsson R:M. \& Kjelleberg S. 1992. - Survival, stress resistance, and alterations in protein expression in the marine $V i$ brio sp. strain $\mathrm{S} 14$ during starvation for different individual nutrients. Appl. Environ. Microbiol., 58 : 55-65.

Parkinson J.S. 1993. - Signal transduction schemes of bacteria. Cell, $73: 857-871$.

Sombille M. \& Billen G. 1983. - A method for determining exoproteolytic activity in natural waters. Limnol. Oceanogr., 28 : 190-193.

Steen H.B., Boye E., Skarstad K., Bloom B., Godal T. \& Mustafa S. 1982. - Applications of flow cytometry on bacteria : cell cycle kinetics, drug effects, and quantitation of antiboy binding. Cytometry, 2 : 249-257.

Talbot V. \& Bianch M. 1997. - Bacterial proteolytic activity in sediments of the Subantarctic Indian Ocean sector. Deep-Sea Res. II, $44: 1069-1084$.

Trevors J.T., Mayfield C.I. \& Inniss W.E. 1982. - Measurement of electron transport system ETS. - activity in soil. Microb. Ecol., 8 : 163-168.

Ward B.B. 1995. - Functional and taxonomic probes for bacteria in the nitrogen cycle. In : Molecular ecology of aquatic microbes. Joint I (Ed.) - NATO ASI Series, Series G : Ecological Sciences, vol 38. Springer-Verlag, Berlin Heidelberg : 73-86.

Wintzingerode F.V., Göbel U.B. \& Stackebrandt E. 1997. - Review : Determination of microbial diversity in environmental samples : pitfalls of PCR-based rRNA analysis. FEMS Microbiol., $21: 229$ 231. 